\title{
Arzt-Patienten-Kontakt
}

\section{Flüchtlingen möglichst offene Fragen stellen}

In Ländern, in denen man Autoritäten nicht widerspricht, sagen Patienten auch "Ja" zum Arzt, wenn sie eigentlich "Nein" meinen. Bei Flüchtlingen kann das Stoff für Fehldiagnosen sein.

Ein tamilischer Flüchtling kommt wegen massiver Rückenbeschwerden zum Orthopäden. Der Arzt verschreibt ihm ein Medikament. Am nächsten Tag geht es dem Patienten deutlich schlechter. Auf die Frage des Arztes „Geht es Ihnen besser?", antwortet er dennoch mit zusammengepressten Lippen: „Ja“. Der Mann kommt aus einem Kulturkreis, in dem man Autoritäten nicht widerspricht. Trotz seiner Schmerzen wollte er dem Arzt nicht das Gefühl geben, seine Behandlung habe versagt.

Um solche Missverständnisse zu vermeiden, sollten Ärzte und Psychotherapeuten in der Arbeit mit Flüchtlingen möglichst mit offenen Fragen arbeiten, empfiehlt Eva van Keuk, Leiterin des psychotherapeutischen Fachbereichs im Psychosozialen Zentrum für Flüchtlinge in Düsseldorf. „Oder Sie geben den Patienten eine Wahlmöglichkeit und fragen: Geht es Ihnen besser oder schlechter?", sagte van Keuk bei einer Fortbildung zur medizinischen Versorgung von Flüchtlingen und Asylsuchenden des Instituts für Qualität im Gesundheitswesen Nordrhein.

\section{Aufklärungspflicht ansprechen}

In vielen Regionen der Welt sei es üblich, dass Ärzte schlimme Diagnosen nicht mitteilen oder zumindest nicht dem $\mathrm{Pa}$ tienten selbst. Auf Basis dieser Erfahrung gehen die Patienten nach dem Gespräch mit einem deutschen Arzt davon aus, dass er ihnen nur einen Teil der Wahrheit gesagt hat. „Es ist hilfreich, wenn sie den Patienten erläutern, dass die Ärzte in Deutschland verpflichtet sind, über alles aufzuklären“, sagte van Keuk.

Um Fehldiagnosen durch sprachliche Missverständnisse zu vermeiden, müssen häufig Dolmetscher zum Einsatz kom- men. Gerade wenn es um psychiatrische Diagnosen geht, sollten niemals die Kinder der Patienten übersetzen, betonte die Diplom-Psychologin.

\section{Auf professionelle Kräfte zurückgreifen}

Ihren Erfahrungen nach können die Familienstrukturen daran zerbrechen, wenn die Kinder ihre Eltern in einer defizitären Rolle erleben. „Die Kinder müssen den Eltern erklären, um was es geht. Das rächt sich spätestens in der Pubertät."

Auch vom Einsatz ehrenamtlicher Sprachmittler rät van Keuk in solchen Situationen ab. „Arbeiten Sie möglichst mit vielen professionellen Kräften“, empfahl auch Dr. Wolf Rommel, niedergelassener Hausarzt und bis vor kurzem Referent bei der Ärztekammer Westfalen-Lippe. Zwar könnten ehrenamtliche Helfer gerade beim Aufbau von Versorgungsstrukturen hilfreich sein. „Auf Dauer arbeitet man aber besser mit Leuten, die ordnungsgemäß vergütet werden“, sagte Rommel.

\section{Unklare Symptome richtig deuten} Dr. Ljiljana Joksimovic, Leitende Oberärztin in der Klinik für Psychosomatische Medizin und Psychotherapie des LVR-Klinikums Düsseldorf, verwies auf eine besondere Herausforderung für die neu zu entwickelnden ambulanten und stationären Behandlungsangebote für Flüchtlinge: die Abklärung unklarer körperlicher Symptome, hinter denen sich schwerwiegende behandlungsbedürftige psychische Erkrankungen verbergen können.

„Wenn diese Erkrankungen unerkannt bleiben, führen sie zu seelischen, körperlichen und sozialen Beeinträchtigungen, welche mit hohem menschli-

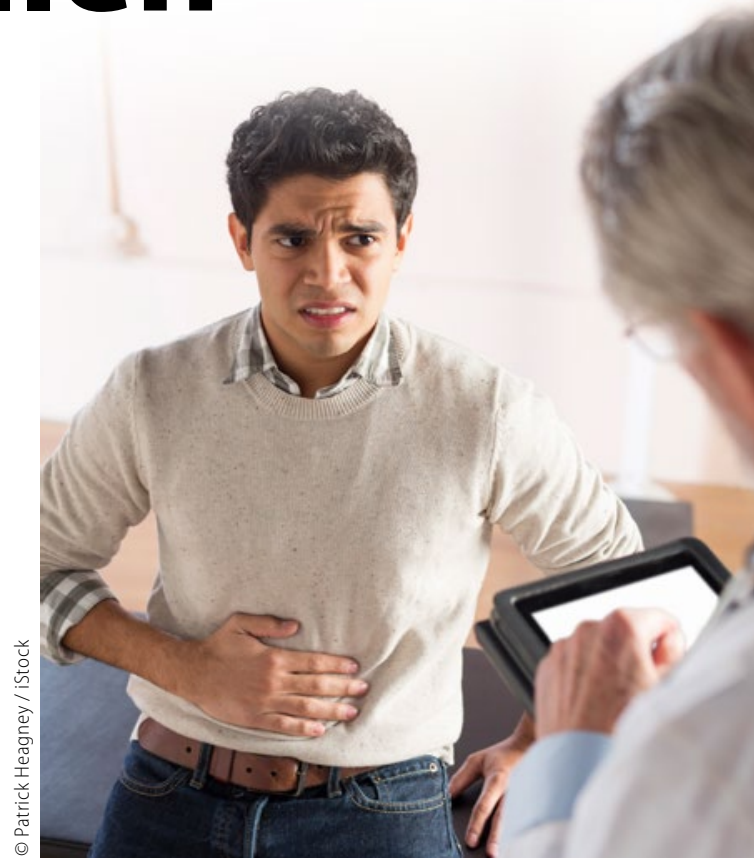

Lassen Sie den Patienten erzählen, um Missverständnisse zu vermeiden.

chen Leid und hohen gesellschaftlichen Kosten einhergehen“, sagte Joksimovic.

\section{Nach Belastungen fragen}

Symptome, die sich somatisch nicht erklären lassen, dürften nicht vorschnell auf soziokulturelle oder religiöse Faktoren zurückgeführt werden, warnte sie. Das sei ein Kunstfehler. „Wir müssen die Erkenntnisse der psychotraumatologischen und psychosomatischen Forschungen einbeziehen, wenn wir Flüchtlinge versorgen", forderte die Ärztin.

Nicht alle Flüchtlinge, die traumatisierenden Belastungen ausgesetzt waren, werden krank. „Rund 30\% haben ein großes Risiko, posttraumatische Belastungsstörungen oder andere Krankheiten zu entwickeln“, sagte Joksimovic. Dennoch sollten Ärzte ihre Patienten immer nach möglichen traumatischen Belastungen fragen. „Die Flüchtlinge äußern sich nicht von sich aus." (Ilse Schlingensiepen)

springermedizin.de; 8. April 2016 